
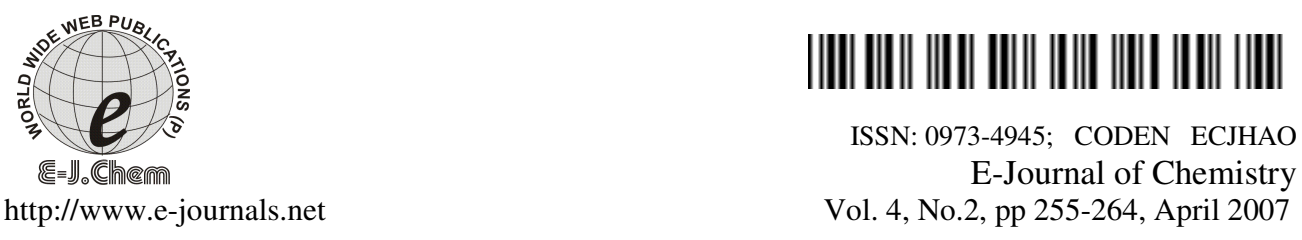

\title{
Studies on the Optical Properties and Surface Morphology of Nickel Phthalocyanine Thin Films
}

\author{
BENNY JOSEPH* and C. S. MENON \\ School of Pure and Applied Physics, \\ Mahatma Gandhi University, \\ Kottayam, 686 560, Kerala State. \\ Email:sjvben@yahoo.com
}

Received 20 September 2006; Accepted 1 November 2006

\begin{abstract}
Thin films of Nickel Phthalocyanine (NiPc) are fabricated at a base pressure of $10^{-5} \mathrm{~m}$.bar using Hind-Hivac thermal evaporation plant. The films are deposited on to glass substrates at various temperatures $318,363,408$ and $458 \mathrm{~K}$. The optical absorption spectra of these thin films are measured. Present studies reveal that the optical band gap energies of NiPc thin films are highly dependent on the substrate temperatures. The structure and surface morphology of the films deposited on glass substrates of temperatures 303 , 363 and $458 \mathrm{~K}$ are studied using X-ray diffractograms and Scanning Electron Micrographs (SEM), show that there is a change in the crystallinity and surface morphology due to change in the substrate temperatures. Full width at half maximum (FWHM) intensity of the diffraction peaks is also found reduced with increasing substrate temperatures. Scanning electron micrographs show that these crystals are fiber like at high substrate temperatures. The optical band gap increases with increase in substrate temperature and is then reduced with fiber-like grains at $408 \mathrm{~K}$. The band gap increases again at $458 \mathrm{~K}$ with full of fiber like grains. Trap energy levels are also observed for these films.
\end{abstract}

Keywords: Nickel Phthalocyanine, Thermal evaporation, Thin film, X-ray diffraction, Scanning electron micrograph.

\section{Introduction}

Organic dyes have attracted attention as novel materials in high-density optical data recording (ODR) media due to their chemical stability, low heat conduction, and diversity of optical properties ${ }^{1}$. Phthalocyanine is an organic semiconductor widely used for sensor 
applications and transistor fabrication, which has excellent stability against heat, light, moisture and oxygen ${ }^{2}$. The physical properties are controlled by traps, which are associated with dislocations, imperfections, grain boundaries and surface topology of film $^{3}$. Nickel phthalocyanine is an organic semiconductor that contains alternate single and double bonds. In this paper the optical band gap of Nickel Phthalocyanine thin films deposited at various substrate temperatures are studied to investigate the changes in the optical properties. The technique of $\mathrm{X}$-ray diffraction (XRD) allows accurate determination of lattice $\mathrm{d}$ spacing, lattice parameters and crystallite size and phase identification ${ }^{4,5,6}$. The optical properties of these materials are dependent on the crystal phases and its structure ${ }^{7}$. The diffractograms obtained are analyzed to study the structure and crystallinity of films. Scanning electron microscopy (SEM) is one of the powerful tools for the investigation of surface topography and micro structural features ${ }^{7,8}$. The present paper used the SEM micrograms to study the surface morphology of the films.

\section{Experimental}

Powdered Nickel phthalocyanine from Aldrich Chemical Co, WI (USA) is used as source material. Glass substrates are cleaned physically and chemically to avoid contaminants from the glass surface. Thoroughly cleaned glass substrates of dimensions $75 \mathrm{~mm} \times 25 \mathrm{~mm} \times 1.35 \mathrm{~mm}$ are used for the evaporation. Resistive heating of NiPc powder from molybdenum boat is carried at the rate of $20 \mathrm{~nm}$ per minute. Thin films of Nickel Phthalocyanine of $400 \mathrm{~nm}$ are prepared at different substrate temperatures on to glass substrate kept in a base pressure of $10^{-5}$ m.bar using a Hind-Hivac thermal evaporation plant. The substrate is placed $20 \mathrm{~cm}$ away from the molybdenum boat. Thickness of the film is measured by Tolansky's multiple beam interference technique ${ }^{9}$. Visible ultra-violet and near infrared absorption spectra of $\mathrm{NiPc}$ thin films on glass substrates of temperatures 318, 363, 408 and $458 \mathrm{~K}$ are recorded using a Shimadzu 160A spectrophotometer and band gaps are investigated.

Thoroughly cleaned glass substrates of dimensions $20 \mathrm{~mm}$ x $20 \mathrm{~mm}$ x $1 \mathrm{~mm}$ are used for film preparation for X-ray diffraction (XRD) studies. The thin films of 400nm thickness are deposited on to the substrates. X-ray diffractograms are recorded for the NiPc thin films coated at the substrate temperatures 303, 363 and 458K using BRUKER D5005 X-ray diffractometer, which is using $\mathrm{Cu} \mathrm{K \alpha}$ radiation of wavelength $1.54059 \AA$. The scanning speed is fixed at 2-degrees/ minute. Full width at half maximum (FWHM) intensity of the diffraction peaks is studied. Pre-cleaned microscopic cover glass substrates of dimensions $16 \mathrm{~mm} \times 16 \mathrm{~mm} \times 0.1 \mathrm{~mm}$ are used for film preparation for SEM analysis. The thin films of $400 \mathrm{~nm}$ thickness on to the substrates deposited at substrate temperatures 303, 363 and $458 \mathrm{~K}$ are used for SEM analysis. The micrograms obtained by using scanning electron microscope LEO 435VP (U K) are analyzed to study surface morphology.

\section{Results and Discussion}

The absorption spectra for Nickel phthalocyanine thin films of thickness 400nm deposited at $318,363,408$ and $458 \mathrm{~K}$ are given in the Fig 1 . The tensile stress produced due to the constraint imposed by the substrate temperature affects the electronic structure and thereby results in new absorption spectra. Anderson et al..$^{10}$ has reported that the central metal atom of the phthalocyanines influences the optical absorption spectrum. Yamashita et al. ${ }^{11}$ have observed that the $\mathrm{Q}$ band absorption shifts towards longer wavelengths when deposition temperatures are increased. Present study shows Q and B bands. It is observed that shift of Q band peak towards longer wavelength above $363 \mathrm{~K}$ and shift towards shorter wavelength side below $363 \mathrm{~K}$. It is also observed that the intensity of absorption maxima observed at $675 \mathrm{~nm}$ become prominent with increasing temperature compared to the prominent peak observed at 
$635 \mathrm{~nm}$ at room temperature, which is due to phase change activity from $\alpha$ to $\beta$ which is in agreement with Jungyoon ${ }^{12}$, Djurisic ${ }^{13}$ and $\mathrm{Xia}^{14}$.

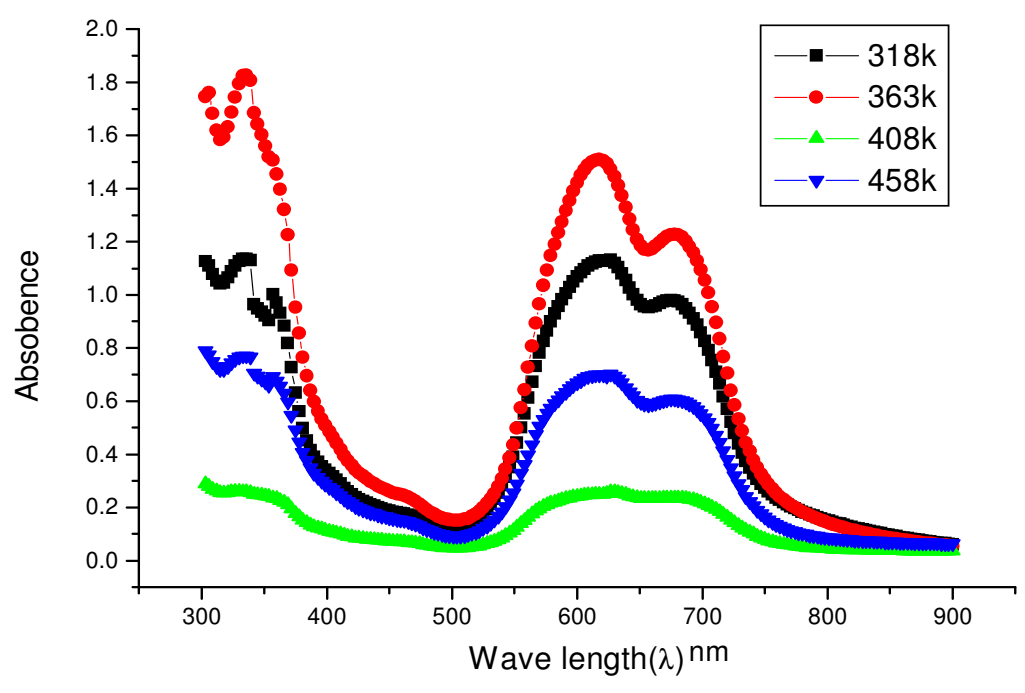

Figure 1. The absorption spectra for Nickel phthalocyanine thin films deposited at $318,363,408$ and $458 \mathrm{~K}$ temperatures.

The absorption at the lower energy side is related to singlet excitons and has been confirmed for many other phthalocyanines ${ }^{15}$. James et al. ${ }^{16}$ observed, all spectra display the existence of strong absorption bands in the range of 400 and $800 \mathrm{~nm}$ caused by the $\pi-\pi^{*}$ transitions of the conjugated macrocycle of $18 \pi$-electrons. The high-energy side of the absorption spectrum shows a sharp absorption edge. The absorbance spectrum shows sharp increase in absorption at wavelength near the absorption edge of the threshold wavelength of the absorbance spectrum, the energy corresponds to this determines the optical band gap of the semiconductor material ${ }^{5}$. The absorption coefficient $\alpha$ is related to the photon energy $\mathrm{h} v$ by $\alpha=\alpha_{0}(\mathrm{~h} v-\mathrm{Eg})^{\mathrm{n}}$, where Eg is the optical band gap. The plots of $\alpha^{2} v s$. hv for the thin films are given in Fig 2 . For allowed transition $\left(\alpha^{2}\right)$ is plotted against energy $(\mathrm{eV})$ to yield a straight line for direct transitions as shown in figure from which the extrapolation of linear portion to $\alpha^{2}=0$ near the absorption edge gives the band gap energy $y^{5}$. The variations in energy gap, trap levels at high temperatures are tabulated in Table-1.

Table 1. The optical band gap energies of Nickel phthalocyanine thin films of thickness $400 \mathrm{~nm}$ deposited at different substrate temperatures.

\begin{tabular}{cccc}
\hline $\begin{array}{c}\text { Substrate } \\
\text { temperature }\end{array}$ & $\begin{array}{c}\text { Trap level 1 } \\
(\mathrm{eV})\end{array}$ & $\begin{array}{c}\text { Trap level 2 } \\
(\mathrm{eV})\end{array}$ & $\begin{array}{c}\text { Energy gap } \\
(\mathrm{eV})\end{array}$ \\
\hline 318 & 1.66 & 1.68 & 3.17 \\
363 & 1.66 & 1.76 & 3.19 \\
408 & 1.43 & 1.43 & 2.79 \\
458 & 1.62 & 1.68 & 3.41 \\
\hline
\end{tabular}




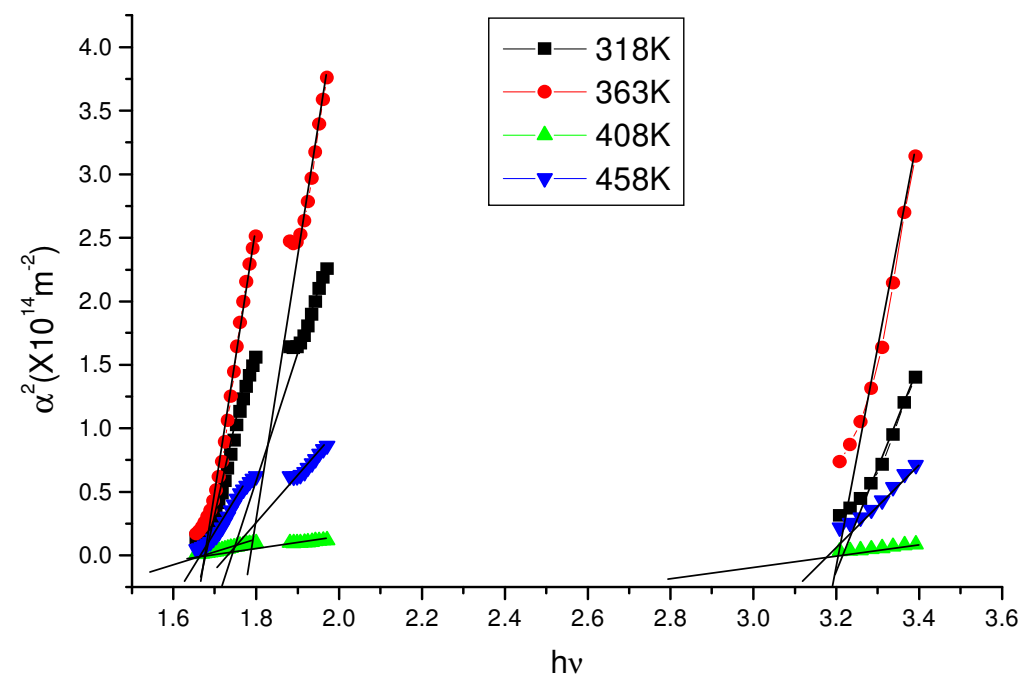

Figure 2. The plot of $\alpha^{2}$ vs hv for NiPc thin films deposited at substrate temperatures $318,363,408$ and $458 \mathrm{~K}$ is given in the.

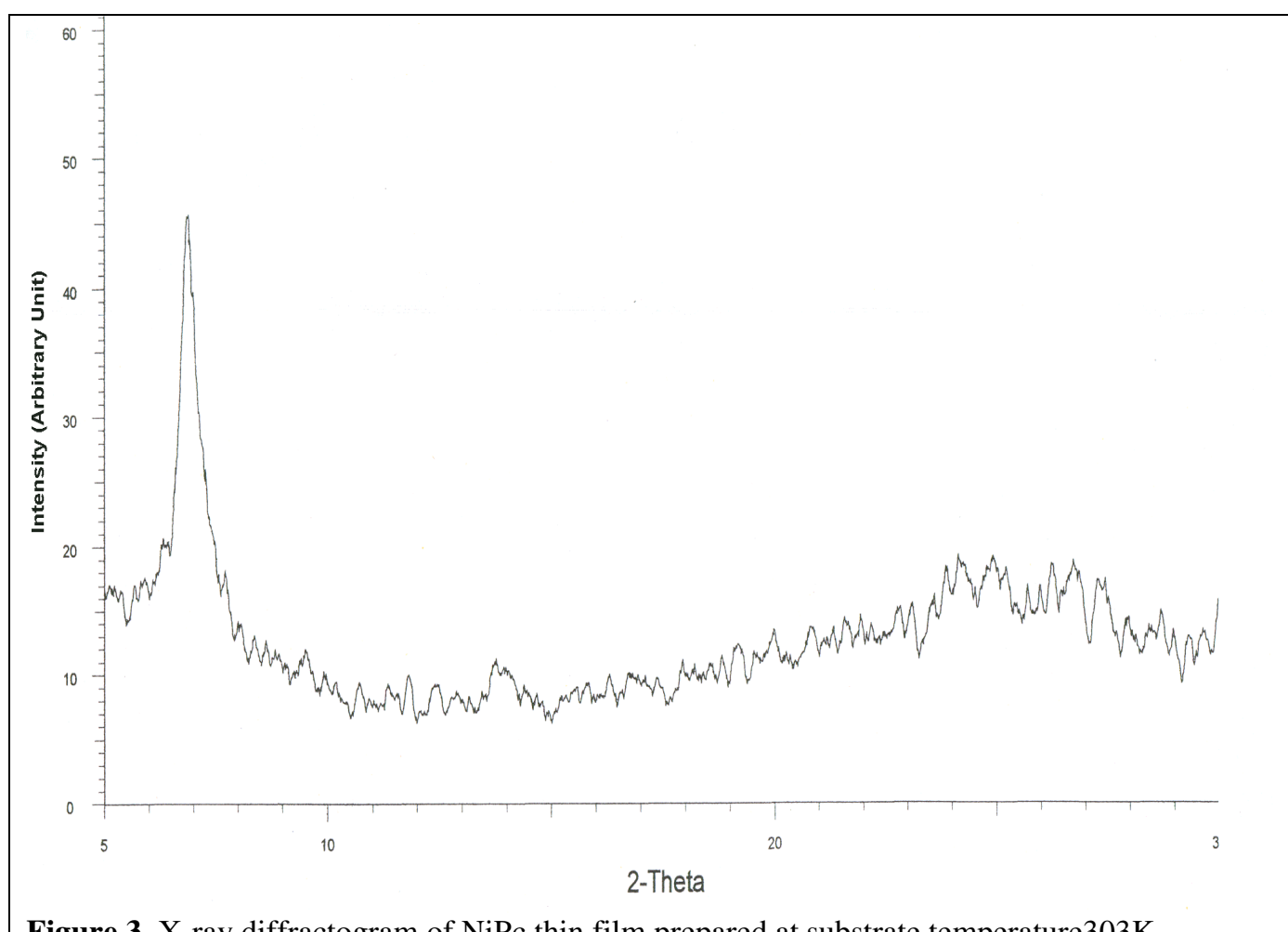

Figure 3. X-ray diffractogram of NiPc thin film prepared at substrate temperature303K 
It is observed that the band gap energy increases initially with substrate temperature and then shows a decrease at $408 \mathrm{~K}$. The band gap energy increases again at $458 \mathrm{~K}$. Agarwal ${ }^{7}$ found that optical band gap for the as deposited thin film is increased on crystallization at low substrate temperature. Garnier ${ }^{17}$ found that an increase of the band gap energy can be expected with lowering of the chain length. The decrease of band gap above $363 \mathrm{~K}$ is due to phase change activity. The film deposited at room temperature is identified to be of $\alpha$-form and the complete phase change is reported around $300^{\circ} \mathrm{C}^{3,12,18}$. Mohammed and Collins ${ }^{19}$ and Fustoss-Wegner ${ }^{20}$ has pointed out that for certain phthalocyanines the phase change occurs in two stages with a well-defined intermediate state. The phase separation could be responsible for the degradation of the optical properties ${ }^{21}$. Campbell and Collins ${ }^{22}$ have shown that the energy band gap of PbPc is narrowed by forming trap levels above the valence band. The trap level slightly increases as the temperature increases and then decreases at temperature 408K. The trap level is again slightly increases at substrate temperature $458 \mathrm{~K}$.

The most common polymorphic crystal phases occur in phthalocyanines are $\alpha$ and $\beta$, that have two crystallite structures. The $\alpha$ phase is metastable and obtained either as a polycrystalline powder or as a thin film deposited on a cold substrate in vacuum ${ }^{23,24}$. The $\beta$ phase can be obtained in single crystal form or as a thin film formed by deposition of phthalocyanine on heated substrates ${ }^{23}$, which are thermodynamically stable ${ }^{24}$. Phase separation could be responsible for the de gradation of the optical properties ${ }^{25}$.

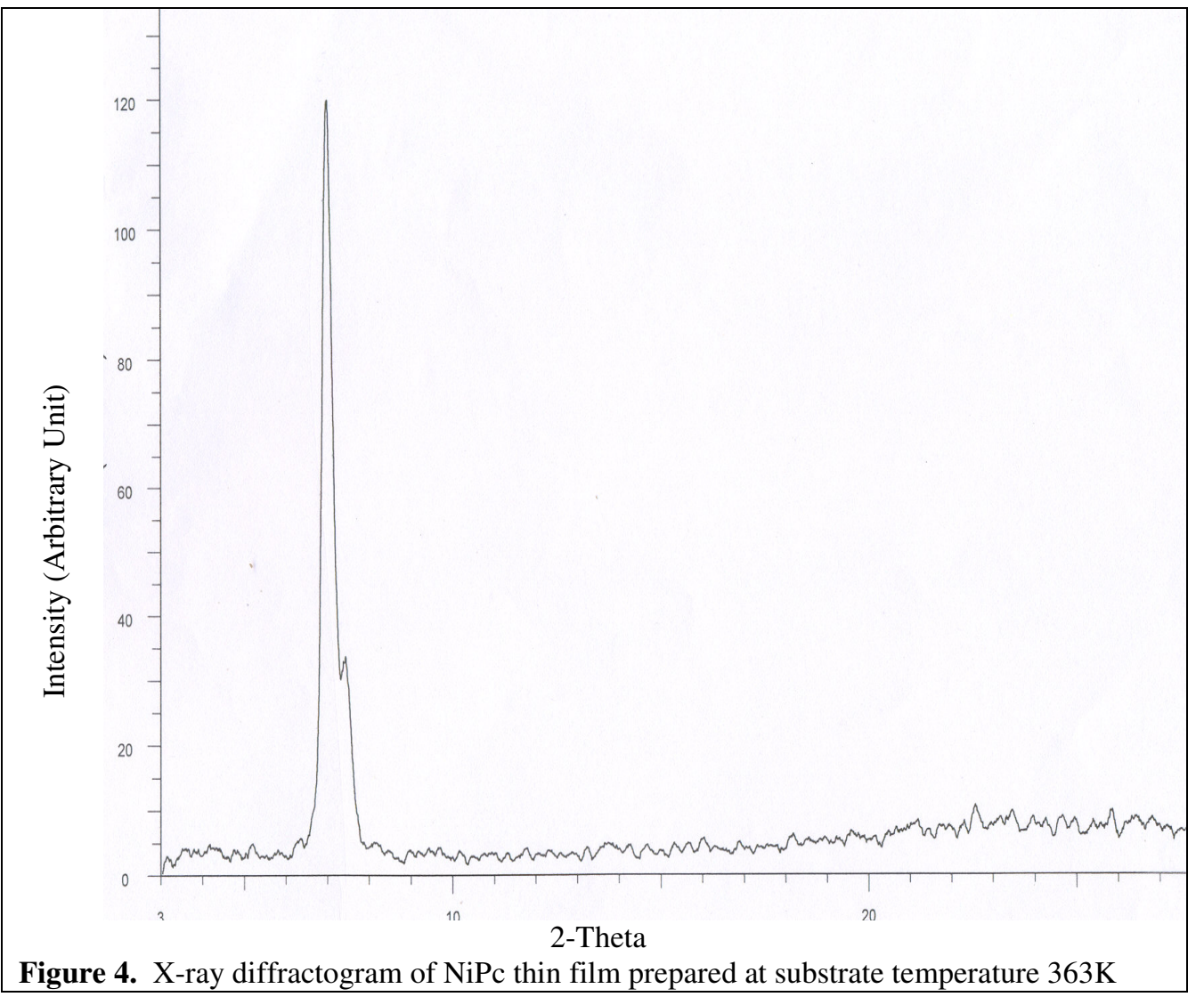




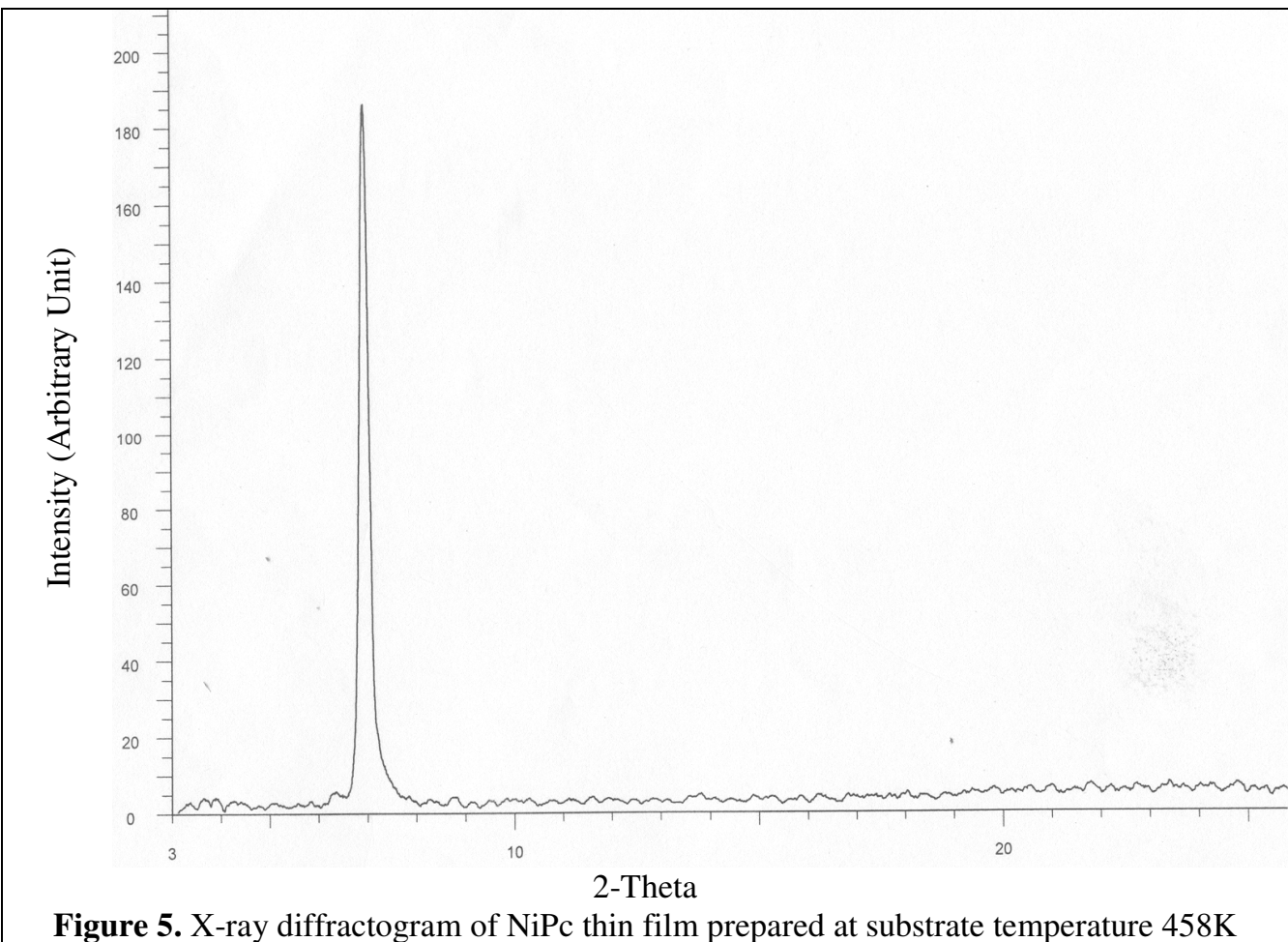

The $\alpha$-form is metastable and can be converted to $\beta$-form by temperature treatments with structures tetragonal and monoclinic respectively ${ }^{9}$. The differences in the $\mathrm{d}$ values are attributed to higher X-ray absorption, sample purity, particle size, preferred orientation and crystal texture ${ }^{6}$. X-ray diffraction pattern of NiPc thin film prepared at substrate temperatures 303, 363 and $458 \mathrm{~K}$ are shown in the figure 3 , figure 4 and figure 5 respectively. XRD pattern indicates that all the samples are not of a single phase $e^{4,5}$. Well-defined diffraction peaks by (001) gives the direction of the preferential orientation in the as deposited film (JCPDS, file no. 11-0744). The preferential orientation is at $2 \theta=6.859^{\circ}$. The spacing between the planes, $d=12.876 \AA$. The XRD pattern of as deposited film shows amorphous nature as reported by many investigators ${ }^{3,9,12}$. The $d$ values obtained from diffractogram are in perfect match with JCPDS record. The diffraction peaks are well matched with the previous observations ${ }^{9}$ and hence the film prepared at room temperature is identified as tetragonal. It can be seen that the intensity and sharpening of the main peak increase with substrate temperature ${ }^{26}$, which shows crystallization of the films. The $\mathrm{d}$ value increases with substrate temperature and the reduction of $2 \theta$ shows a phase change activity ${ }^{9}$. The difference between $\alpha$ - and $\beta$-phases are attributed to the tilt angle of $b$-axis of the unit cell ${ }^{12}$. The optical studies also support the above observations. The increased crystallinity is attributed to the destruction of pseudomorphic layers present in the film upon high substrate temperature. Table 2 shows the full widths at half maximum (FWHM) intensity of preferential orientation, which shows that the FWHM are reduced with increasing substrate temperatures, which represents better lattice quality ${ }^{27}$.

The scanning electron micrographs of thin films deposited at 303, 363 and $458 \mathrm{~K}$ are shown in the Figures 6, 7 and 8 respectively. The film morphology changes for the elevated substrate temperatures. At low substrate temperature $(303 \mathrm{~K})$ fine, uniform and smooth grain morphology is observed are amorphous ${ }^{7,8}$. The polycrystalline nature of thermally deposited 
$\alpha-\mathrm{NiPc}$ is confirmed by the electron micrographs. As substrate temperature is increased to $363 \mathrm{~K}$ some fiber like grains are found with some out standing clusters (white dots) without appreciable increase in grain size. This phenomenon indicates that the film preferentially grows normal to the surface ${ }^{3}$. The fiber like grains are parallel to the substrate surface and the out standing clusters are reduced due to the high substrate temperature of $458 \mathrm{~K}$.

Table 2. Variation of FWHM of preferential orientation with substrate temperatures for the NiPc thin films.

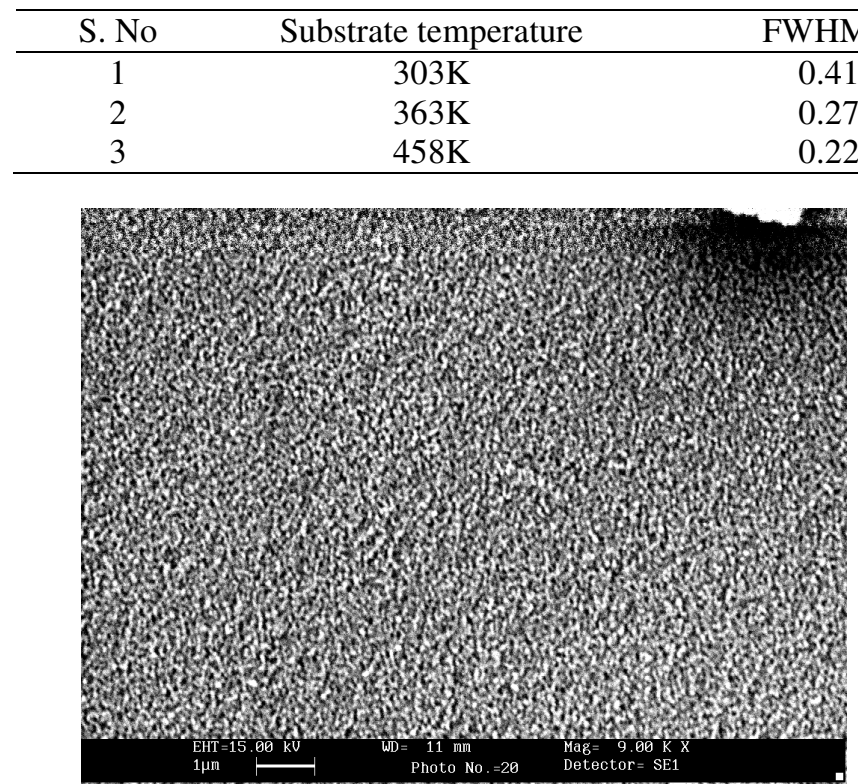

Figure 6. The scanning electron micrographs of thin film deposited at $303 \mathrm{~K}$ substrate temperatures.

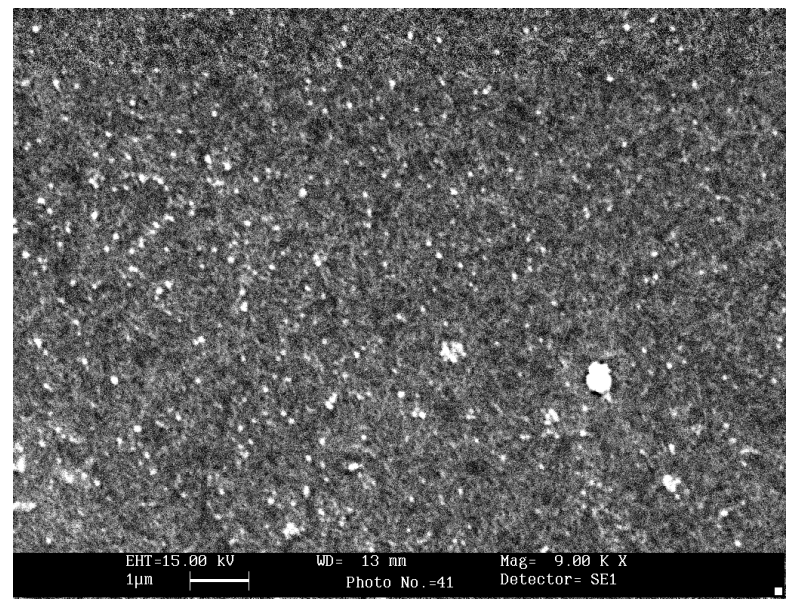

Figure 7. The scanning electron micrographs of thin film deposited at 363K-substrate temperature. 
As substrate temperature increases to $458 \mathrm{~K}$, the crystallinity of the films and density of the grains, parallel to the substrate surface are found increased like $\mathrm{CuPc}^{28}$. It is also observed that the lengths of fiber like grains are reduced slightly, which is against the commonly accepted view and may be due to any unnoticed ambient or fusing of grains which enhanced surface roughness. Band gap at this temperature is found to increase. The reduction in grain size can either be due to partial resublimation, recrystallisation or phase transformation from $\alpha$ to $\beta$. Shrinking of grains at high temperature has been observed earlier ${ }^{7}$. The stacking axis of the fiber at $458 \mathrm{~K}$ is parallel to the substrate surface as in previous observations ${ }^{3}$. The grain size is measured from the micrographs ${ }^{26}$. The growth conditions have a strong influence on the properties of $\mathrm{NiPc}$ thin films like $\mathrm{CuPc}$ films ${ }^{3}$.

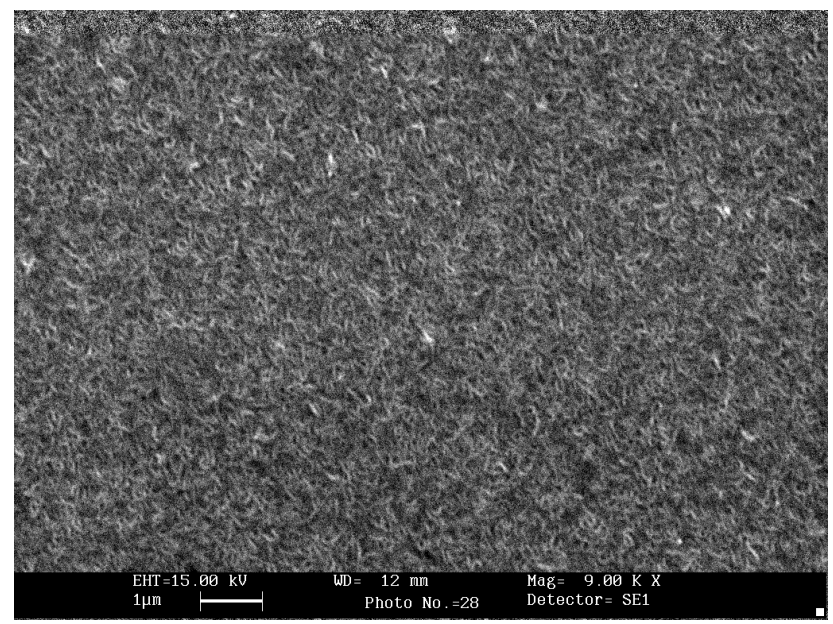

Figure 8. The scanning electron micrographs of thin film deposited at $458 \mathrm{~K}$-substrate temperature.

The crystal morphology and molecular orientation change with substrate temperature ${ }^{1}$. The average grain size increases as temperature increases. Gas sensitivity depends both up on material parameters, such as the crystal phase of the material and film parameters, such as the surface topography of the film ${ }^{8}$. These structural characterizations confirm that the substrate temperature allows us to control the structural organization of nickel phthalocyanine molecules in their solid state, from which a large improvement of the properties can be expected. Table-3 shows that the grain size increased by increasing the substrate temperature. The band gaps, trap levels and activation energies are influenced by the grain size ${ }^{20}$. Grain boundaries act as barriers for the cross transport of charge carriers. The morphology of these films, analyzed by the scanning electron microscopy, has also confirmed the appearance of polycrystallites and increasing grain size when substrate temperature is raised ${ }^{5}$.

Table 3 Variation of grain size with substrate temperatures for the NiPc thin films

\begin{tabular}{cccc}
\hline S.No & $\begin{array}{c}\text { Sub temp, } \\
(\mathrm{K})\end{array}$ & $\begin{array}{c}\text { Grain length, } \\
(\mathrm{nm})\end{array}$ & $\begin{array}{c}\text { Grain breadth, } \\
(\mathrm{nm})\end{array}$ \\
\hline 1 & 303 & 83.0 & 80 \\
2 & 363 & 650.0 & 50 \\
3 & 458 & 500.0 & 50 \\
\hline
\end{tabular}


The difference in grain size may be attributed by the difference in temperature. This is caused due to the nucleating behavior of the substance ${ }^{4}$.

\section{Conclusions}

The optical properties of NiPc films are controlled by energy band gap and the trap levels present in the forbidden energy gap, which are found to be dependent on substrate temperatures. Since the interactions of nickel phthalocyanine molecules are of Vander Waals type, the rearrangements of molecule alter the energy band gap between valence band and conduction band. Rearrangements of molecules are taking place with different substrate temperatures. A sharp change in band gap is observed for film prepared at $458 K$. Diffraction peaks obtained for the films prepared at higher substrate temperatures are sharp and that is due to the increased crystallinity. The NiPc films prepared at room temperature are tetragonal. The difference in d values shows phase change activity with different substrate temperatures. The change in grain size, optical band gap and trap levels with substrate temperature shows that the properties of $\mathrm{NiPc}$ is controlled by its structure and morphology. The average grain sizes are found increased with increase in substrate temperatures

\section{References}

1. Hisao Yanagi, Takashi Kouzeki, and Michio Ashda. J. Appl. Phya. 1992, 71(10), 5146.

2. Sadaoka Y, Jones T A and Gopel W, Sens. Actuators, 1990, B1, 148.

3. Yuh-Lang Lee, Wen-Ching Tsai, and Jer-Ru Maa, Applied surface science 2001,173, 352.

4. Rout S K, Panigrahi S and Bera J, J. bull. matter. 2005, 28, 275.

5. Raji P, Sanjeeviraja C and Ramachandran K, Bull. Matter. Sci., 2005, 28(3), 233.

6. Hassan A K and Gould R D, Phys. Stat. Solid (a), 1992, 91,132.

7. Gargi Agarwal, Reddy G B, J. Materials Science: Materials in Electronics, 2005, 16, 21.

8. Campbell D and R A. Collins, Phys. stat. sol. (a), 1995, 152, 431.

9. Narayanan Unni K N and Menon C S, Materials Letters, 2000, 45, 326.

10. Anderson J S, Bradbrook E F, Cook A H and Linstead R P, J. Chem. soc., 1938, Part II, 1151.

11. Yamashita A, Maruno T, Hayami T, J. Phys. Chem., 1993, 97, 4567.

12. Jungyoon E, Sunmi Kim, Eunju Lim, Kiejin Lee, Deokjoon Cha, Barry Friedman, Applied Surface Science, 2003, 205, 274.

13. Djurisic A B, Kwong C Y, Lau T W, Guo W L, Li E.H, Liu Z T, Kwok H S, Lam L S $\mathrm{M}$ and Chan W K Optics Communicatioms, 2002, 205,155.

14. Xia H, Nogami M, Opt. Mater., 2000, 15, 93.

15. Verzimakha Y A I., Kovalchuk A V, Hamann C, Kurik M V, and Muller M, Phys. Stat. Sol.(a), 1982, 74, KI09.

16. James S A, Ray A K, and Silver J, Phys. Stat. Sol. (a), 1992, 129, 435.

17. Francis Garnier, Chemical Physics, 1998, 227, 253.

18. Shihub S I and Gould R D, Thin Solid Films, 1996, 290, 390.

19. Collins R.A. and Mohammed K.A, Themochimica Acta, 1987,109, 397.

20. Fostoss-Wegner M, Themochimica Acta, 1978, 23, 93.

21. Herrera M, Gonzalez D, Hopkinson M and Navaretti P, J. Semicond. Sci. Technol. 2004, 19, 813.

22. Campbell D and Collins R A, Thin Solid Films, 1995, 261, 311. 
23. Allen Twarowski, J. Chem. Phys., 1982, 77(9), 4698.

24. Karl F.Schoch Jr., James Greggi Jr. and Thomas A Temofonte., J. Vac. Sci. Technol. A, 1988, 6, 155.

25. Herrera M, Gonzalez D, Hopkinson M, Navaretti P, Gutierrez M, Liu H Y and Garcia R., Semicond. Sci. Technol., 2004, 19, 813.

26. Collins R A and Mohammed K A, Thin Solid Flims, 1986, 145,133.

27. Hu X.J, Li R. B, Shen H S, Dai Y B and He X C, Carbon, 2004, 42, 1501.

28. Dogo S, Germain J P, Maleysson C and Pauly A, Thin Solid Films, 1992, 219, 244. 


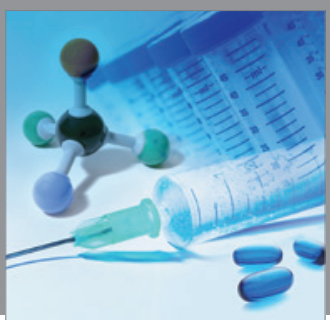

International Journal of

Medicinal Chemistry

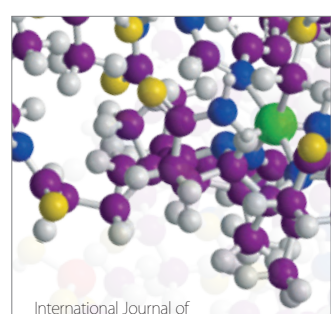

Carbohydrate Chemistry

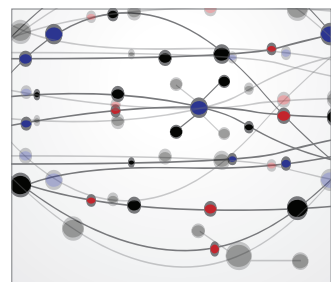

The Scientific World Journal
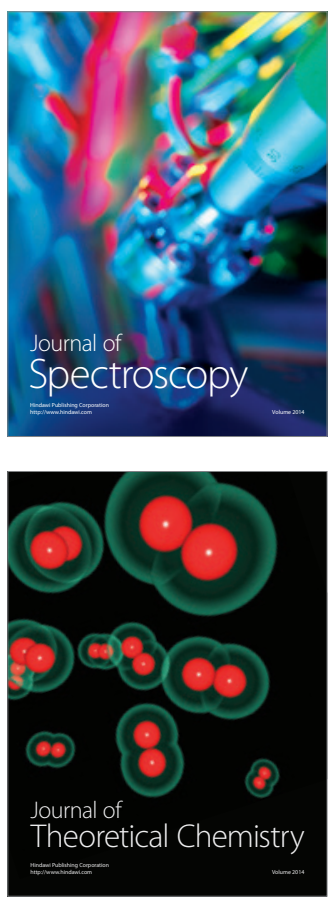
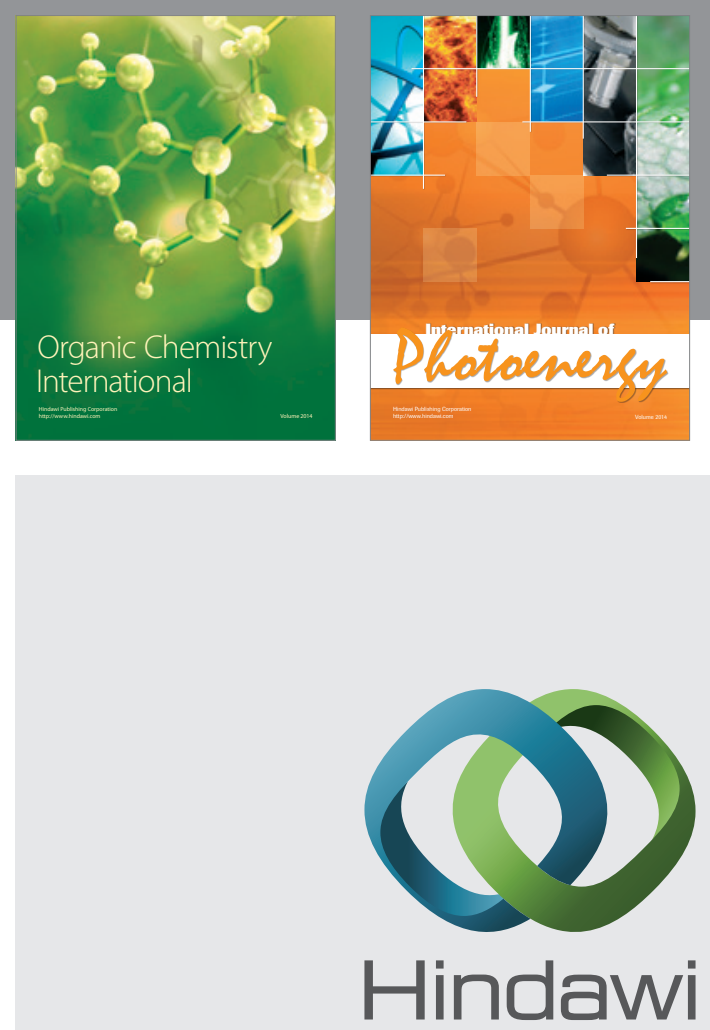

Submit your manuscripts at

http://www.hindawi.com
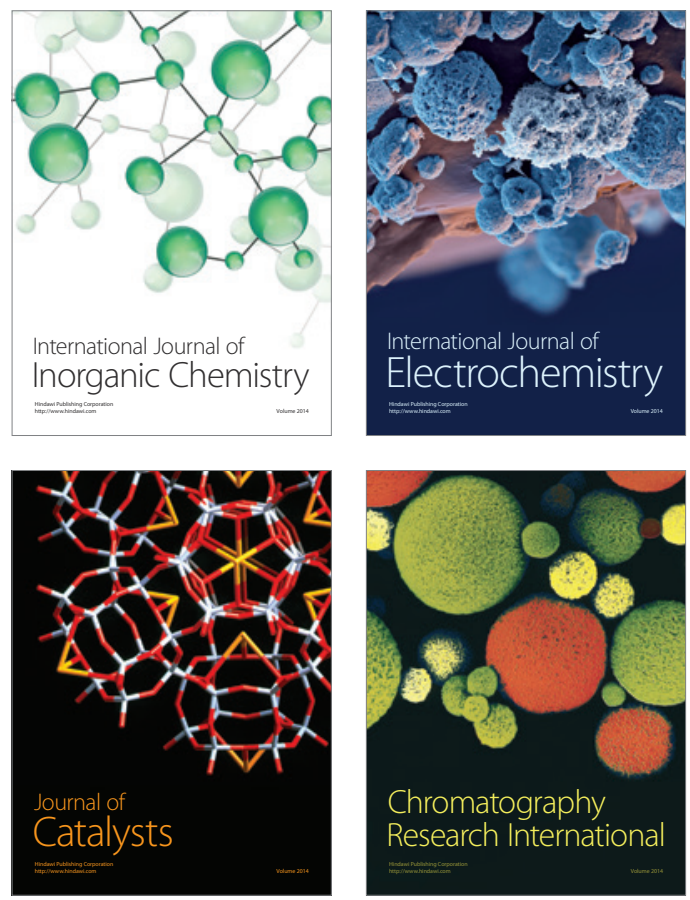
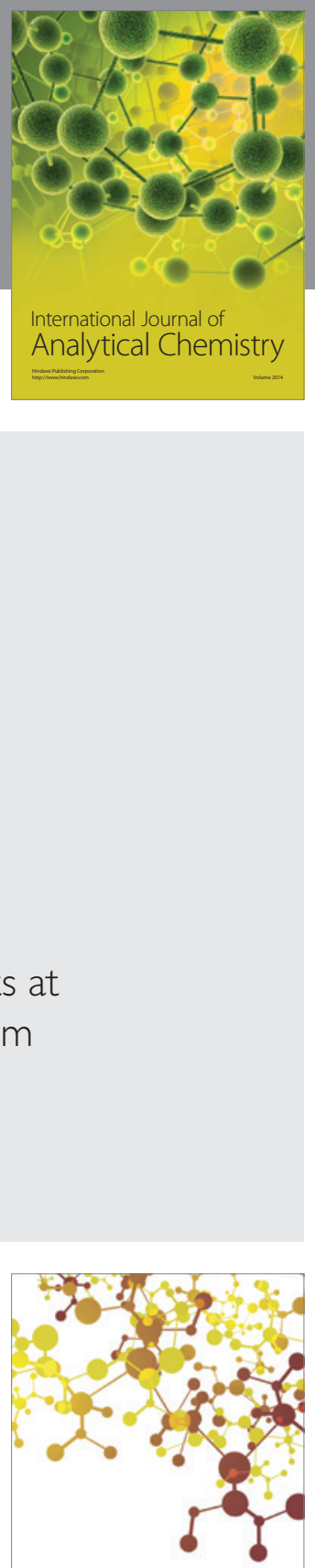

Journal of

Applied Chemistry
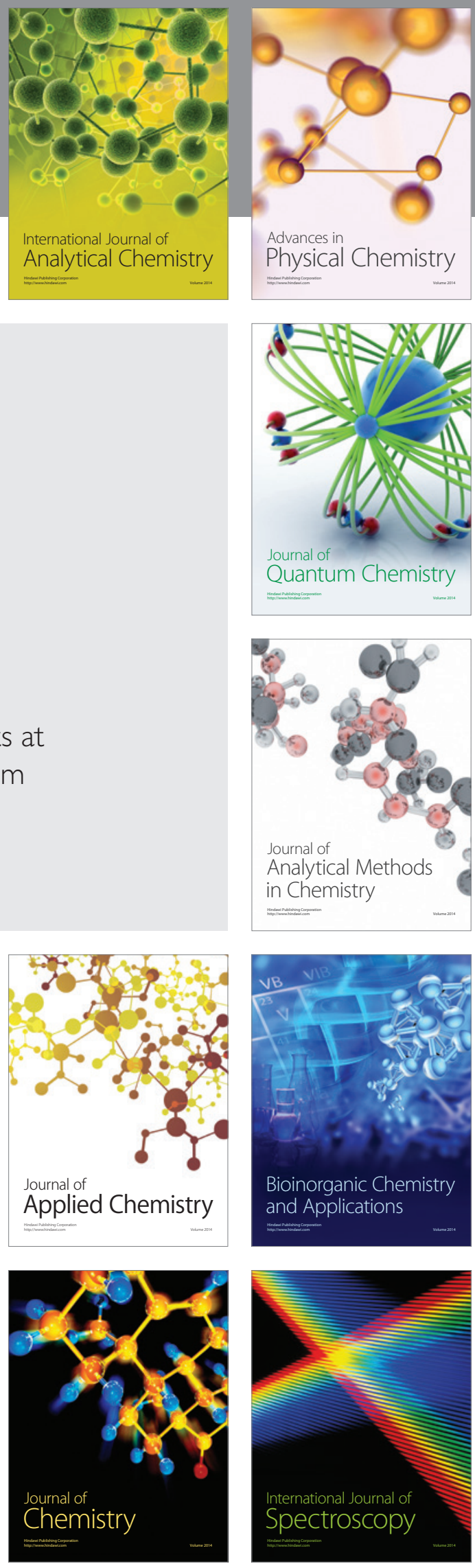